\title{
Detrimental Effects of Multiple Mutations in Position 240 of Fusarium graminearum $\boldsymbol{\beta}_{2}$-Tubulin
}

\author{
Yuanye Zhu, Yuanshuai Zhang, Zongzhe He, Yabing Duan, Yanjun Li, Jianxin Wang, and Mingguo Zhou ${ }^{\dagger}$ \\ College of Plant Protection, Nanjing Agricultural University, Nanjing, 210095, China; and State \& Local Joint Engineering Research Center of \\ Green Pesticide Invention and Application, Nanjing, 210095, China \\ Accepted for publication 24 April 2020.
}

ABSTRACT

\begin{abstract}
Fusarium graminearum causes Fusarium head blight (FHB), a destructive disease of cereal crops worldwide. Carbendazim (methylbenzimidazol-2ylcarbamate $[\mathrm{MBC}]$ ) is widely used for controlling FHB. A previous study showed that the F240L mutation in the $\beta_{2}$-tubulin of $F$. graminearum $\left(F g \beta_{2}\right.$ tubulin) confers hypersensitivity to $\mathrm{MBC}$. Whether the substitution of phenylalanine by other amino acids in position 240 of the $F g \beta_{2}$-tubulin gene also confers hypersensitivity to MBC is unknown. Moreover, the biological fitness of these mutants is poorly understood. In this study, we substituted position 240 of $F g \beta_{2}$-tubulin with other amino acids. We found that the F240A, F240E, F240I, and F240Y mutations in $F g \beta_{2^{-}}$ tubulin could also confer $F$. graminearum hypersensitivity to MBC, although the effective concentration resulting in $50 \%$ inhibition $\left(\mathrm{EC}_{50}\right)$ differed among the mutations. The F240G mutation, in contrast, decreased the sensitivity to MBC. In addition, a molecular docking assay indicated that the binding affinity between $F g \beta_{2}$-tubulin and MBC
\end{abstract}

were increased by the F240A, F240E, F240I, and F240Y mutations but decreased by the F240G mutation. All mutants had normal conidial morphology, but the growth rates and pathogenicity of the F240A, F240E, F240G, F240I, and F240Y mutants were significantly decreased. Moreover, the F240A and F240G mutants produced twisted hyphae. In addition, microtubules were sparse and rarely observed in $\beta_{2}$ F240A-EGFP, $\beta_{2}$ F240E-EGFP, and $\beta_{2}$ F240G-EGFP. These results indicate that position 240 (phenylalanine) is not only vital to the function of $\mathrm{Fg}_{2}$-tubulin but also plays an important role in regulating the sensitivity of $F$. graminearum to MBC. Any mutation in this site would be detrimental to survival.

Keywords: biological fitness, disease control and pest management, $\mathrm{Fg} \mathrm{\beta}_{2}$ tubulin, Fusarium graminearum, genetics and resistance, mutation, position 240
Fusarium graminearum is a destructive pathogen that causes Fusarium head blight (FHB), a devastating disease of wheat and other cereals. In addition to causing yield losses, FHB infected plants produce grains that are often contaminated with mycotoxin, which poses a serious threat to the health of humans and livestock (Bai and Shaner 2004; Chen et al. 2018).

Because cultivars with complete resistance to FHB are lacking, farmers usually control the disease by applying chemical fungicides (Jia et al. 2019). Although the fungicide carbendazim (methylbenzimidazol-2-ylcarbamate [MBC]) has been widely used to control FHB in China, after 40 years of its application, resistant strains have been detected in the field (Chen et al. 2009). MBC binds to $\beta$-tubulin and disrupts microtubule dynamics (Davidse 1986), and resistance to $\mathrm{MBC}$ is conferred by mutations in the $\beta$-tubulin gene (Davidse and Flach 1977).

Microtubules are polymers of $\alpha / \beta$-tubulin heterodimers that interact with each other in a head-to-tail fashion to form protofilaments; the protofilaments associate laterally and form hollow, cylindrical polymers (Nogales 2000). Microtubules can switch spontaneously between a growing and shrinking status, a character termed "dynamic instability" (Aher and Akhmanova 2018; Vitre et al. 2008). Microtubules are essential for many intracellular activities such as cell division, material transportation, and cell

†Corresponding author: M. Zhou; mgzhou@njau.edu.cn

Funding: This research was supported by The Key Program of National Natural Science Foundation of China (31730072).

*The $\boldsymbol{e}$-Xtra logo stands for "electronic extra" and indicates that two supplementary figures and one supplementary table are published online.

The author(s) declare no conflict of interest.

C 2020 The American Phytopathological Society shape maintenance (Huffaker et al. 1988; Niwa et al. 2013). The importance of these activities makes microtubules a useful target for anticancer drugs and pesticides like paclitaxel, vinblastine, MBC, etc. (Davidse and Flach 1977; Steinmetz and Prota 2018), and mutations in the tubulin gene confer resistance to these compounds (Hari et al. 2003; Yarden and Katan 1993). Moreover, mutations in the tubulin gene may also affect the function of tubulin. For example, mutations in both $\alpha$ - and $\beta$-tubulin genes affect microtubule dynamics and disrupt microtubule functions, which indicates that these residues are vital to the structure and functions of tubulins (Ti et al. 2016; Tischfield et al. 2010).

We previously reported that leucine in position 240 (L240) of $\beta$-tubulin is conserved among the fungal plant pathogens: Botrytis cinerea, Colletotrichum gloeosporioides, Monilinia laxa, Sclerotinia sclerotiorum, and Tapesia yallundae. In F. graminearum, however a phenylalanine occurs in position 240 of $\beta_{2}$-tubulin (F240 of $F g \beta_{2}$-tubulin) (Zhu et al. 2018). According to two other studies, an L240F mutation in the $\beta$-tubulin gene of M. laxa and T. yallundae confers resistance to a benzimidazole fungicide (Albertini et al. 1999; Ma et al. 2005). In F. graminearum, an F240L mutation in $\beta_{2^{-}}$ tubulin gene confers hypersensitivity to benzimidazole fungicide and an L240F mutation in $\beta$-tubulin gene of $B$. cinerea confers resistance to benzimidazole fungicides ( $\mathrm{Zhu}$ et al. 2018). In addition, $F$. graminearum is less sensitive to $\mathrm{MBC}$ than $B$. cinerea, C. gloeosporioides, or S. sclerotiorum (Li et al. 2002). These results suggest that the F240 of $F g \beta_{2}$-tubulin is unique and explains why MBC sensitivity is lower for $F$. graminearum than for other plant pathogens. It remains unclear, however, whether substitution of phenylalanine with amino acids other than leucine at position 240 of $F g \beta_{2}$-tubulin also confers hypersensitivity to MBC or affects the biological fitness of $F$. graminearum.

In this study, we substituted phenylalanine with amino acids at position 240 of $F g \beta_{2}$-tubulin by site-directed mutagenesis and assessed the biological fitness and MBC sensitivities of the 
resulting mutants. Interestingly, all mutants except F240L had a decreased growth rate and virulence. Some mutants produced twisted hyphae. Additionally, all mutants except F240G mutant had increased sensitivity to MBC. Using EGFP-tagging and molecular docking, we explored the mechanisms of variation of $\mathrm{MBC}$ sensitivity and phenotypes including decreased growth rate, twisted hyphae, decreased pathogenicity. In addition to increasing our understanding of the importance of position 240 of $\beta_{2}$-tubulin in $F$. graminearum, our results could also be useful for optimizing or developing new tubulin-binding fungicides.

\section{MATERIALS AND METHODS}

Strains, cultural condition, and fungicides. All strains used in this study are listed in Table 1 . The wild-type $F$. graminearum $\mathrm{PH}-1$ was used as the parent strain for site-directed mutagenesis (Zhao et al. 2015). Conidia and mycelia were produced at $25^{\circ} \mathrm{C}$ in mung bean liquid (MBL) medium and yeast extract peptone dextrose (YEPD) medium, respectively (Zhu et al. 2018). For sensitivity assays, all strains were grown on potato dextrose agar (PDA) containing $\mathrm{MBC}$ at $25^{\circ} \mathrm{C}$. $\mathrm{MBC}$ was dissolved in $0.1 \mathrm{M} \mathrm{HCl}$ at $10 \mathrm{mg} / \mathrm{ml}$ as a stock solution, which was subsequently diluted as indicated later in the Methods section (Chen et al. 2009).

Site-directed mutagenesis and protoplast transformation. The F240A, F240E, F240G, F240I, F240L, and F240Y mutants and $\beta_{2}$-tubulin complementary mutant $\left(\Delta \beta_{2}-C\right)$ were constructed by site-directed mutagenesis and gene replacement strategy as previously described (Zhu et al. 2018). The amino acids alanine, glutamic acid, glycine, isoleucine, tyrosine, and phenylalanine differ in their side chains, which facilitated our study of position 240. Alanine has been widely used to investigate the residues of many proteins (Reijo et al. 1994). Glutamic acid contains a polar group. Glycine lacks a side chain. Isoleucine is an isomer of leucine, while tyrosine contains a benzene ring that is similar to that of phenylalanine. The wild-type and mutant $F g \beta_{2}$-tubulin DNA fragments were transformed into protoplasts of the $\mathrm{PH}-1 \beta_{2}$-tubulin deletion mutant strain $\Delta \beta_{2}$, and the transformants were identified by polymerase chain reaction (PCR), sequencing, and Southern blot (Supplementary Fig. S1). Protoplasts of $\Delta \beta_{2}$ were prepared and transformed as previously described (Zheng et al. 2014). Primers used in this study are listed in Supplementary Table S1.

Conidial and hyphal morphology, and conidia production. For examination of the conidial morphology, all strains were inoculated into MBL liquid medium for 3 days, respectively. For examination of the hyphal morphology, all strains were inoculated on PDA plates for $24 \mathrm{~h}$. Spores and hyphae were photographed using an Olympus IX-71 inverted microscope. To compare the number of septa per conidium between position 240 mutants and the wild-type strain PH-1, fresh spores were stained with calcofluor white (CFW) and examined by fluorescence microscopy (Zhang et al. 2016). For each strain, 200 spores were counted. The numbers of conidia produced by position 240 mutants and the wild-type strain PH- 1 were assessed and compared as previously described (Zheng et al. 2014).

Pathogenicity assay. A coleoptile infection assay was conducted to compare the virulence of position 240 mutant strains with that of the wild-type strain $\mathrm{PH}-1$ as previously described (Zhang et al. 2012). In brief, Huaimai 33 wheat seeds were placed in culture dishes and placed in a growth chamber with lights. After 3 days, the top 2 to $3 \mathrm{~mm}$ of each coleoptile was removed, and the wound was inoculated with $2.5 \mu \mathrm{l}$ of fresh conidia $\left(10^{6} / \mathrm{ml}\right)$ harvested from the MBL medium. Water-inoculated coleoptiles were used as a control. After 7 to 10 days, the infected coleoptiles were photographed, and the lengths of disease lesions were measured. At least 20 wheat seedlings were measured for each strain. This experiment was conducted three independent times.

Construction of an $F \boldsymbol{g} \beta_{2}$-tubulin-C-EGFP strain and confocal microscopy. To visualize $\beta_{2}$-tubulin, we labeled wildtype and mutant $\beta_{2}$-tubulin with enhanced green fluorescent protein (EGFP) at the COOH-terminal (Zhu et al. 2018). The schematic for vector construction is described in Supplementary Figure S2. First, three fragments were obtained by PCR: a $2.8-\mathrm{kb}$ fragment containing $1.1-\mathrm{kb}$ upstream fragment and $1.7-\mathrm{kb}$ coding sequence (CDS) of $F g \beta_{2}$-tubulin gene, which were amplified from the DNA template of wild-type strain $\mathrm{PH}-1$ by primers $\mathrm{P} 1$ and $\mathrm{P} 13$; a $0.7-\mathrm{kb}$ EGFP CDS, which was amplified from plasmid PYF11 (Bruno et al. 2004) by primers P14 and P15; and a 1.0-kb downstream fragment, which was amplified from the DNA template of wild-type strain PH-1. Second, the EGFP and downstream fragments were mixed at a 1:1 molar ratio and used as a template for double-joint PCR to generate a 1.7-kb EGFP-downstream fragment (Zheng et al. 2014). Finally, the upstream- $F g \beta_{2} \mathrm{CDS}$ and EGFP-downstream fragments were mixed at a 1:1 molar ratio and used as a template for doublejoint PCR to generate the final vector, which was used for transforming the protoplasts of strain $\Delta \beta_{2}$. The position 240 mutant strains were labeled with EGFP using the same approach. The transformants were identified by PCR, sequencing, and fluorescence observation (Supplementary Fig. S2).

Microtubules were photographed with a LEICA TCS SP8 confocal laser-scanning microscope as previously described (Zhu et al. 2018).

Growth rates and $\mathrm{MBC}$ sensitivities. For measurement of growth rates, $5 \mathrm{~mm}$ mycelial plugs taken from the margin of a 3day-old colony of each strain and placed on PDA plates (one plug per plate, three plates per strain). The plates were kept at $25^{\circ} \mathrm{C}$. The colony diameters were measured after 3 days. This experiment was conducted three independent times.

Radial growth was used to determine the sensitivities of position 240 mutants to MBC. Because the position 240 mutants differ in

TABLE 1. Strains and mutants of Fusarium graminearum used in this study

\begin{tabular}{lll}
\hline Strains and mutants & \multicolumn{1}{c}{ Genotype } & Reference \\
\hline PH-1 & Wild-type strain of $F$. graminearum & Zhang et al. (2012) \\
$\Delta \beta_{2}$ & $F g \beta_{2}$-tubulin deletion mutant in PH-1 genetic background & This study \\
$\Delta \beta_{2}$-C & $\beta_{2}$-tubulin complementary mutant & This study \\
F240A-1 and F240A-2 & $\beta_{2}$ F240A mutant generated from $\Delta \beta_{2}$ & This study \\
F240E-1 and F240E-2 & $\beta_{2}$ F240E mutant generated from $\Delta \beta_{2}$ & This study \\
F240G-1 and F240G-2 & $\beta_{2}$ F240G mutant generated from $\Delta \beta_{2}$ & This study \\
F240I-1 and F240I-2 & $\beta_{2}$ F240I mutant generated from $\Delta \beta_{2}$ & This study \\
F240L-1 and F240L-2 & $\beta_{2}$ F240L mutant generated from $\Delta \beta_{2}$ & This study \\
F240Y-1 and F240Y-2 & $\beta_{2}$ F240Y mutant generated from $\Delta \beta_{2}$ & This study \\
$\beta_{2}$-EGFP & $\beta_{2}$-tubulin-C-EGFP fusion mutant generated from $\Delta \beta_{2}$ & This study \\
$\beta_{2}$ F240A-EGFP & $\beta_{2}$ F240A-EGFP fusion mutant generated from $\Delta \beta_{2}$ & This study \\
$\beta_{2}$ F240E-EGFP & $\beta_{2}$ F240E-EGFP fusion mutant generated from $\Delta \beta_{2}$ & This study \\
$\beta_{2}$ F240G-EGFP & $\beta_{2}$ F240G-EGFP fusion mutant generated from $\Delta \beta_{2}$ & This study \\
$\beta_{2}$ F240I-EGFP & $\beta_{2}$ F240I-EGFP fusion mutant generated from $\Delta \beta_{2}$ & This study \\
$\beta_{2}$ F240L-EGFP & $\beta_{2}$ F240L-EGFP fusion mutant generated from $\Delta \beta_{2}$ & This study \\
$\beta_{2}$ F240Y-EGFP & $\beta_{2}$ F240Y-EGFP fusion mutant generated from $\Delta \beta_{2}$ & This study \\
\hline
\end{tabular}


growth rate, the timing of radial growth measurement differed for the mutants. Colony diameters were measured after 3 days for PH-1, $\Delta \beta_{2}-\mathrm{C}, \mathrm{F} 240 \mathrm{I}$, and F240L; after 5 days for F240E, and F240Y; and after 10 days for F240A and F240G. The dose response curve and effective concentration resulting in $50 \%$ inhibition $\left(\mathrm{EC}_{50}\right)$ value for each mutant were calculated using a Data Processing System (DPS) computer program (Hangzhou Reifeng Information Technology Ltd.). This experiment was conducted three independent times.

The MBC concentration used to assess sensitivity to MBC were selected based on preliminary experiments that indicated the general sensitivities of the position 240 mutants. MBC was added to PDA plates at $0,0.2,0.4,0.6,0.8,1.0$, and $1.2 \mu \mathrm{g} / \mathrm{ml}$ for PH1 and $\Delta \beta_{2}$-C; at $0,0.1,0.2,0.3,0.4,0.5,0.6$, and $0.8 \mu \mathrm{g} / \mathrm{ml}$ for F240Y; at 0 , $0.02,0.04,0.06,0.08,0.1$, and $0.12 \mu \mathrm{g} / \mathrm{ml}$ for F240E, F240I, and F240L; at $0,0.05,0.1,0.15,0.2$, and $0.25 \mu \mathrm{g} / \mathrm{ml}$ for F240A; and at 0 , $0.5,0.75,1.0,1.25,1.5,1.75$, and $2.0 \mu \mathrm{g} / \mathrm{ml}$ for F240G.

RNA extraction and quantitative real-time PCR. According to a previous study, an altered expression level of $F g \beta_{2}$-tubulin could affect sensitivity to MBC (Gu et al. 2019). To exclude the possibility that mutation at position 240 could affect the expression of $F g \beta_{2}$-tubulin, we assessed the relative expression level of $F g \beta_{2^{-}}$ tubulin of all mutants. In brief, five 5-mm-diameter mycelial plugs
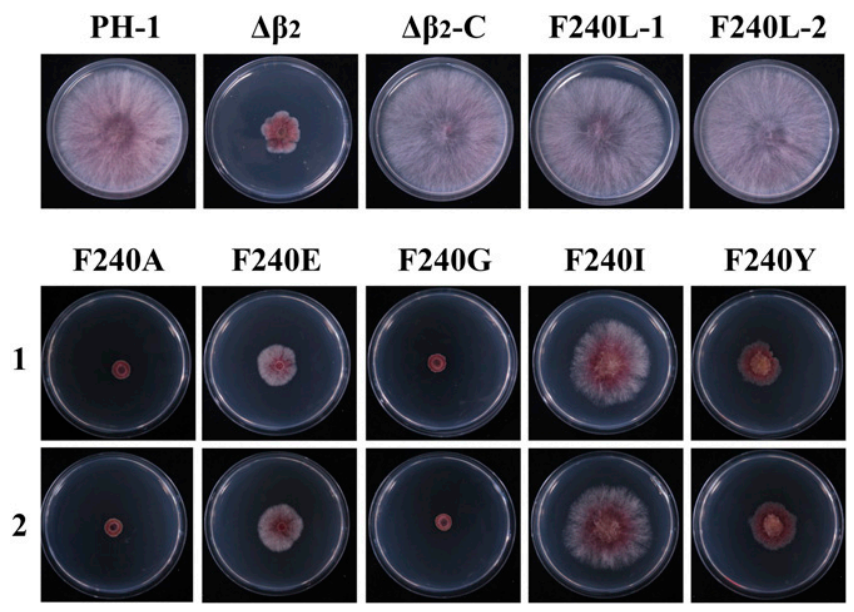

F240E

F240G

F240I

F240Y
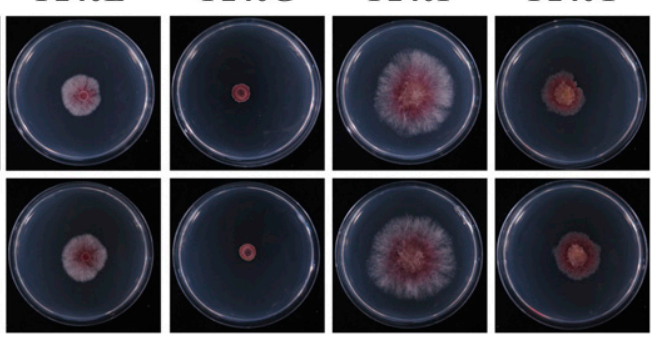

Fig. 1. Colonial morphology of wild-type strain PH-1, $F g \beta_{2}$-tubulin deletion mutant $\Delta \beta_{2}$, the complemented mutant $\Delta \beta_{2}-\mathrm{C}$, and position 240 mutants. All strains were photographed after growing on potato dextrose agar plants for 3 days at $25^{\circ} \mathrm{C}$.

TABLE 2. Sensitivities of Fusarium graminearum strains to methylbenzimidazol-2-ylcarbamate (MBC) and hyphal growth rates ${ }^{\mathrm{x}}$

\begin{tabular}{lcc}
\hline Strain & $\mathrm{EC}_{50}(\mu \mathrm{g} / \mathrm{ml})^{\mathrm{y}}$ & Growth rate $(\mathrm{cm} / \text { day })^{\mathrm{z}}$ \\
\hline PH-1 & $0.4871 \pm 0.0090 \mathrm{~b}$ & $2.69 \pm 0.19 \mathrm{a}$ \\
$\Delta \beta_{2}$ & $0.1020 \pm 0.0171 \mathrm{def}$ & $0.35 \pm 0.09 \mathrm{e}$ \\
$\Delta \beta_{2}-\mathrm{C}$ & $0.4637 \pm 0.0136 \mathrm{~b}$ & $2.55 \pm 0.09 \mathrm{a}$ \\
F240A-1 & $0.1127 \pm 0.0038 \mathrm{de}$ & $0.27 \pm 0.03 \mathrm{e}$ \\
F240A-2 & $0.1158 \pm 0.0192 \mathrm{~d}$ & $0.26 \pm 0.02 \mathrm{e}$ \\
F240E-1 & $0.0437 \pm 0.0009 \mathrm{ef}$ & $1.26 \pm 0.11 \mathrm{c}$ \\
F240E-2 & $0.0420 \pm 0.0028 \mathrm{ef}$ & $1.25 \pm 0.09 \mathrm{c}$ \\
F240G-1 & $0.8681 \pm 0.1635 \mathrm{a}$ & $0.23 \pm 0.04 \mathrm{e}$ \\
F240G-2 & $0.8311 \pm 0.1476 \mathrm{a}$ & $0.22 \pm 0.02 \mathrm{e}$ \\
F240I-1 & $0.0347 \pm 0.0028 \mathrm{f}$ & $2.19 \pm 0.09 \mathrm{~b}$ \\
F240I-2 & $0.0350 \pm 0.0037 \mathrm{f}$ & $2.09 \pm 0.18 \mathrm{~b}$ \\
F240L-1 & $0.0524 \pm 0.0014 \mathrm{def}$ & $2.71 \pm 0.16 \mathrm{a}$ \\
F240L-2 & $0.0529 \pm 0.0059 \mathrm{def}$ & $2.66 \pm 0.13 \mathrm{a}$ \\
F240Y-1 & $0.2350 \pm 0.0162 \mathrm{c}$ & $0.94 \pm 0.04 \mathrm{~d}$ \\
F240Y-2 & $0.2413 \pm 0.0208 \mathrm{c}$ & $0.96 \pm 0.04 \mathrm{~d}$
\end{tabular}

$x$ Values are means and standard deviations. Means in the table followed by the same letter are not significantly different $(P<0.05)$.

${ }^{y}$ MBC concentration that resulted in 50\% mycelial growth inhibition.

${ }^{\mathrm{z}}$ Growth rate was measured after incubation of three replicates for 3 days. were taken from the margin of a 3-day-old colony (on PDA plates) of all mutant strains; the plugs were transferred into $100 \mathrm{ml}$ of YEPD medium, which was incubated at $25^{\circ} \mathrm{C}$ with agitation (175 rpm). After $36 \mathrm{~h}$, the fresh mycelia were harvested and washed with deionized water. Total RNA was extracted following the instructions of the Total RNA Extraction Kit (Tiangen, China, DP419). First-strand cDNA was synthesized by HiScript II Reverse transcription (Vazyme, Nanjing, China, R223-01). The ChamQ SYBR qPCR Master Mix (Vazyme, Nanjing, China, Q311-02/03) was used for quantitative real-time PCR (qRT-PCR), which was conducted with the CFX Connect Real-Time System (Bio-Rad) and with the following program: $95^{\circ} \mathrm{C}$ for $30 \mathrm{~s}$; followed by 40 cycles at $95^{\circ} \mathrm{C}$ for $10 \mathrm{~s}$ and $60^{\circ} \mathrm{C}$ for $30 \mathrm{~s}$; and melting curve step of at $95^{\circ} \mathrm{C}$ for $15 \mathrm{~s}$ and $60^{\circ} \mathrm{C}$ for $60 \mathrm{~s}$, followed by 71 cycles of gradual increase in temperature $(13 \mathrm{~s} / \mathrm{cycle})$ from 60 to $95^{\circ} \mathrm{C}$. The primers used for qRT-PCR analysis are listed in Supplementary Table S1. The expression of the $F g \beta_{2}$-tubulin gene in each sample was normalized to that of the Fgactin gene.

Homology modeling and molecular docking. To investigate the effects of mutations at position 240 of $F g \beta_{2}$-tubulin on MBC sensitivity, we conducted molecular docking assays. The homology models of wild-type and mutant $F g \beta_{2}$-tubulins were generated by the online server I-TASSER (https://zhanglab.ccmb. med.umich.edu), which combines the methods of threading, ab initio modeling and structural refinement (Roy et al. 2010; Zhang 2008). All homology models were constructed based on the same remark parents (Protein Date Bank ID: 3hke_D, 1tvk_B, 3cb2_A, $4 \mathrm{u} 3 \mathrm{j} \_\mathrm{B}$, and 2btq_A). Molecular docking between $F g \beta_{2}$-tubulin and MBC was performed using AutoDock Tools in MGL Tools software (version 1.5.6) with semiflexible docking (Zhang et al. 2019). Docking parameters used the Lamarck genetic algorithm (LGA); the docking box size was set to $40 \AA * 40 \AA * 40 \AA$ and the conformation was set as flexible for MBC but rigid for $\mathrm{Fg}_{2}$-tubulin. The software generated 256 times results, and we chose the lowest binding free energy conformation as the stable conformation.

Statistical analysis. Data processing was performed using DPS software. The results for hyphal growth rate, relative $F g \beta_{2^{-}}$ tubulin gene expression level, conidia production, MBC sensitivity, and lesion length (pathogenicity) were expressed as means \pm standard deviations from three independent experiments.

\section{RESULTS}

Construction of position 240 mutant strains. To study the importance of position 240 of $F g \beta_{2}$-tubulin, we used site-directed mutagenesis in order to substitute phenylalanine in position 240 with the following six amino acids: alanine (Ala, A), glutamic acid (Glu, E), glycine (Gly, G), isoleucine (Ile, I), leucine, (Leu, L), and tyrosine (Tyr, Y). After using sequencing, PCR amplification, and Southern blotting to confirm that the mutations occurred as designed (Supplementary Fig. S1). We compared the phenotypes of these mutants with those of the $F g \beta_{2}$-tubulin deletion mutant $\Delta \beta_{2}$, the complemented $F g \beta_{2}$-tubulin deletion mutant $\Delta \beta_{2}-\mathrm{C}$, and the wild-type strain PH-1 as described in the next sections.

Vegetative growth, morphology, pathogenicity, and relative expression level of the $\mathrm{Fg} \beta_{2}$-tubulin gene of the position 240 mutants. Growth rates were slower for F240A, F240E, F240G, F240I, and F240Y mutants than for the wild type or the F240L mutant (Fig. 1 and Table 2). Hyphal and conidial morphology did not differ among F240E, F240I, F240L, and F240Y mutants and the wild-type strain PH-1 (Figs. 2 and 3). F240A and F240G mutants, in contrast, had twisted hyphae, which were similar to those of $\Delta \beta_{2}$ (Fig. 2). The CFW assay indicated that $30 \%$ of the spores of mutants F240A and F240G were aseptate (Figs. 3 and 4A). In addition, the mean numbers of conidia produced by F240A, F240E, F240G, F240I, F240L, and F240Y mutants were significantly increased to those produced by the parent strain $\Delta \beta_{2}$, but were 
slightly less than the wild-type strain PH-1, and $\Delta \beta_{2}-\mathrm{C}$ (Fig. 4B). Lesions on the coleoptiles of wheat were significantly smaller after inoculation with mutants F240A, F240E, F240G, F240I, and F240Y than after incubation with the wild-type, F240L, or $\Delta \beta_{2}-\mathrm{C}$ (Fig. 5). The mean expression levels of $F g \beta_{2}$-tubulin relative to that in the wild-type strain PH-1 were slightly higher in F240A-1, F240A-2, F240E-1, F240G-1, F240I-1, F240I-2, F240L-1, and F240Y-1; similar in F240Y-2; and slightly lower in $\Delta \beta_{2}-\mathrm{C}$, F240G-2, and F240L-2 (Fig. 6). These differences, however, were not statistically significant.

Microtubule polymerization of position 240 mutants. The microtubule network was dense and easily observed in $\beta_{2^{-}}$

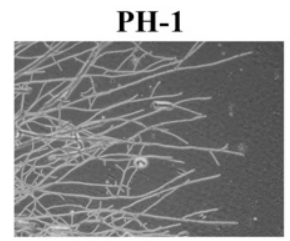

F240A

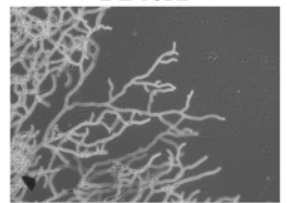

F240I

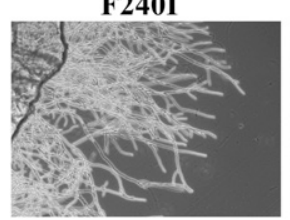

$\Delta \beta 2$

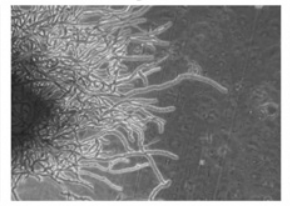

F240E

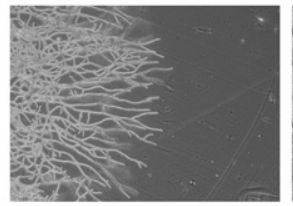

F240L

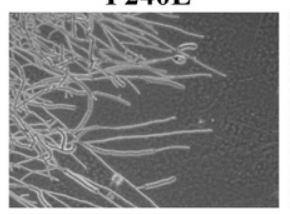

$\Delta \beta 2-C$

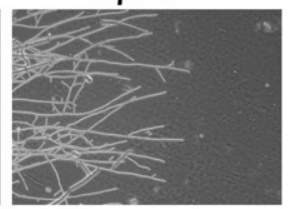

F240G

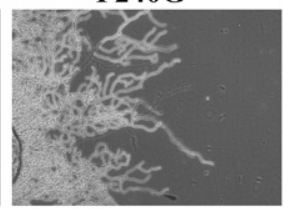

F240Y

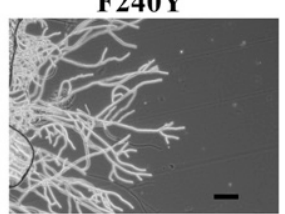

Fig. 2. Hyphal morphology of wild-type strain $\mathrm{PH}-1, \mathrm{Fg} \beta_{2}$-tubulin deletion mutant $\Delta \beta_{2}$, the complemented mutant $\Delta \beta_{2}-\mathrm{C}$, and position 240 mutants. Hyphal morphology was photographed after strains were incubated on potato dextrose agar (PDA) plants for $24 \mathrm{~h}$ at $25^{\circ} \mathrm{C}$. Bar $=20 \mu \mathrm{m}$.
EGFP, $\beta_{2}$ F240I-EGFP, $\beta_{2}$ F240L-EGFP, and $\beta_{2}$ F240Y-EGFP, but was sparse and difficult to detect in $\beta_{2}$ F240A-EGFP, $\beta_{2}$ F240EEGFP, and $\beta_{2}$ F240G-EGFP (Fig. 7).

MBC sensitivity of position 240 mutants. F240A, F240E, $\mathrm{F} 240 \mathrm{I}$, and $\mathrm{F} 240 \mathrm{Y}$ were hypersensitive to $\mathrm{MBC}$ with $\mathrm{EC}_{50}$ values of $0.1127 \pm 0.0038,0.0437 \pm 0.0009,0.0347 \pm 0.0028$, and $0.2350 \pm$ $0.0162 \mu \mathrm{g} / \mathrm{ml}$, respectively. F240G had a slightly decreased sensitivity to $\mathrm{MBC}$ with an $\mathrm{EC}_{50}$ value of $0.8681 \pm 0.1635 \mu \mathrm{g} / \mathrm{ml}$ (Table 2).

Molecular docking between $\boldsymbol{F g} \boldsymbol{\beta}_{2}$-tubulin and MBC. A molecular docking assay indicated that $\mathrm{MBC}$ binds to the binding pocket of $F g \beta_{2}$-tubulin, which is in the vicinity of the previously reported residues: Phe167, Glu198, and Phe200 (Qiu et al. 2012). The F240A mutant formed a strong H-bond with MBC at Ile258, and the binding free energy was decreased from -5.81 to $-5.95 \mathrm{kcal} /$ mol, although only one H-bond was formed. The F240E mutant formed an H-bond with MBC at Val236 and Glu198, and the binding free energy was decreased from -5.81 to $-6.31 \mathrm{kcal} / \mathrm{mol}$. The F240G mutant formed only one H-bond with MBC at Tyr222, and the binding free energy was increased from -5.81 to $-5.73 \mathrm{kcal} /$ mol. The F240I mutant formed an H-bond with MBC at Arg156, Ser196, and Asp197; these three bonds decreased the binding free energy up to $-6.34 \mathrm{kcal} / \mathrm{mol}$. The F240L mutant formed an H-bond with MBC at Asp197, and at Pro261; these two bonds decreased the binding free energy from -5.81 to $-6.27 \mathrm{kcal} / \mathrm{mol}$. The F240Y mutant formed an $\mathrm{H}$-bond with MBC at Cys12, which decreased the binding free energy from -5.81 to $-5.94 \mathrm{kcal} / \mathrm{mol}$ (Fig. 8 and Table 3 ).

\section{DISCUSSION}

In this study, we investigated the functions of position 240 of $F g \beta_{2}$-tubulin by substituting Phe at position 240 with six other amino acids including Ala, Glu, Gly, Ile, Leu, and Tyr. The differences in the side chains of these six amino acids facilitated our investigation of the importance of position 240 in the $\beta_{2}$-tubulin gene of $F$. graminearum. We found that the mutants F240A, F240E, and F240G had slower growth rates than the wild type, or the F240I and F240L mutants (Fig. 1 and Table 2) and that the F240A, F240E, and F240G mutants produced twisted hyphae (Fig. 2). A previous
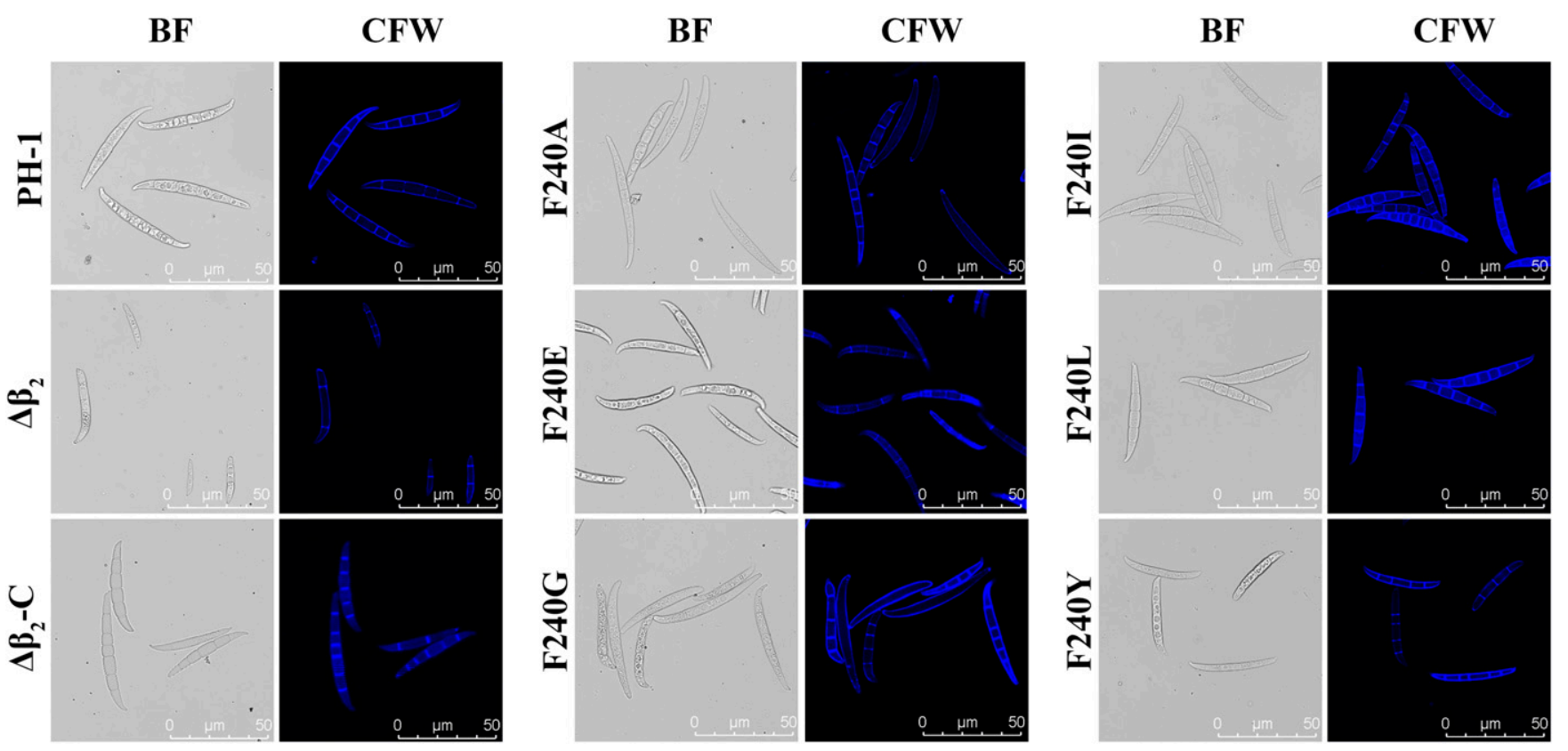

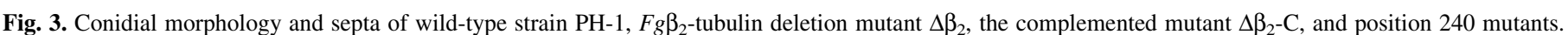

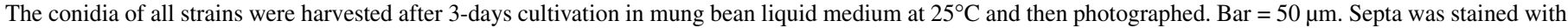
calcofluor white and then photographed using a confocal laser-scanning microscope. 
study showed that $F g \beta_{2}$-tubulin is essential for vegetative growth and that a decrease in the expression level of $F g \beta_{2}$-tubulin by RNA interference or the disruption of the $F g \beta_{2}$-tubulin gene reduced the growth rate of $F$. graminearum (Gu et al. 2019; Liu et al. 2013). However, we found no significant difference in the expression level of the $\mathrm{Fg}_{2}$-tubulin gene among the mutants and the wild-type strain, which suggested that the slower growth rate or abnormal hyphal morphology was not due to a reduced expression level of the $F g \beta_{2}$-tubulin gene (Fig. 6). Therefore, these phenotypes could only be produced by a functional defect in tubulin caused by position240 mutation. Our observations are consistent with other studies. Reijo et al. (1994), for example, reported that a substitution with alanine from position 249 to 252 of yeast $\beta$-tubulin generated a recessive-lethal phenotype. The L240I mutation of $\beta$-tubulin in human leukemia cells located at $\mathrm{H} 7$ and $\mathrm{H} 8$ helixes affected the longitudinal interaction between two $\alpha$-/ $\beta$-tubulin heterodimers and decreased microtubule stability (Kavallaris et al. 1997). The R262C mutation in TUB3B (neuron-specific $\beta$-tubulin isotype III) of human nerve cells reduced microtubule polymerization (Tischfield et al. 2010). Consistent with these earlier reports, we found that the $\beta_{2}$-tubulin-EGFP was diffusely distributed and barely polymerized into microtubules in the hyphae of F240A, F240E, and F240G mutants (Fig. 7). This suggested that the decreased growth rate and abnormal hyphae of these mutants resulted from disrupted microtubule polymerization and dynamics. Together, earlier studies and current results indicate that a mutation near the region of position 240 of $\beta$-tubulin may decrease the stability of microtubules, which is essential for producing viable cells with normal
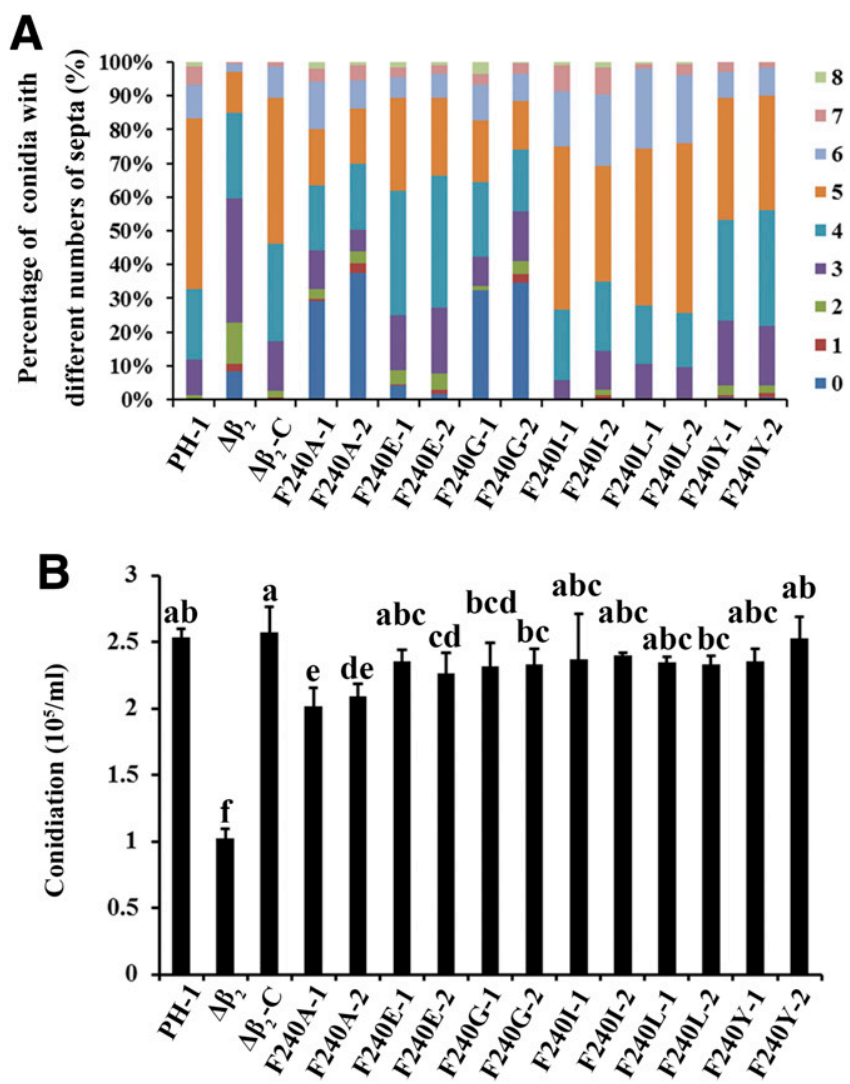

Fig. 4. Septa numbers and conidial productions of wild-type strain $\mathrm{PH}-1$, $F g \beta_{2}$-tubulin deletion mutant $\Delta \beta_{2}$, the complemented mutant $\Delta \beta_{2}-\mathrm{C}$, and position 240 mutants. A, Percentage of conidia with different numbers of septa. For each strain, 200 spores were counted. B, Conidial productions of all strains. Conidial production was measured by hemacytometer after 3 days incubation in mung bean liquid medium. Means of the column with the same letter are not significantly different $(P<0.05)$.
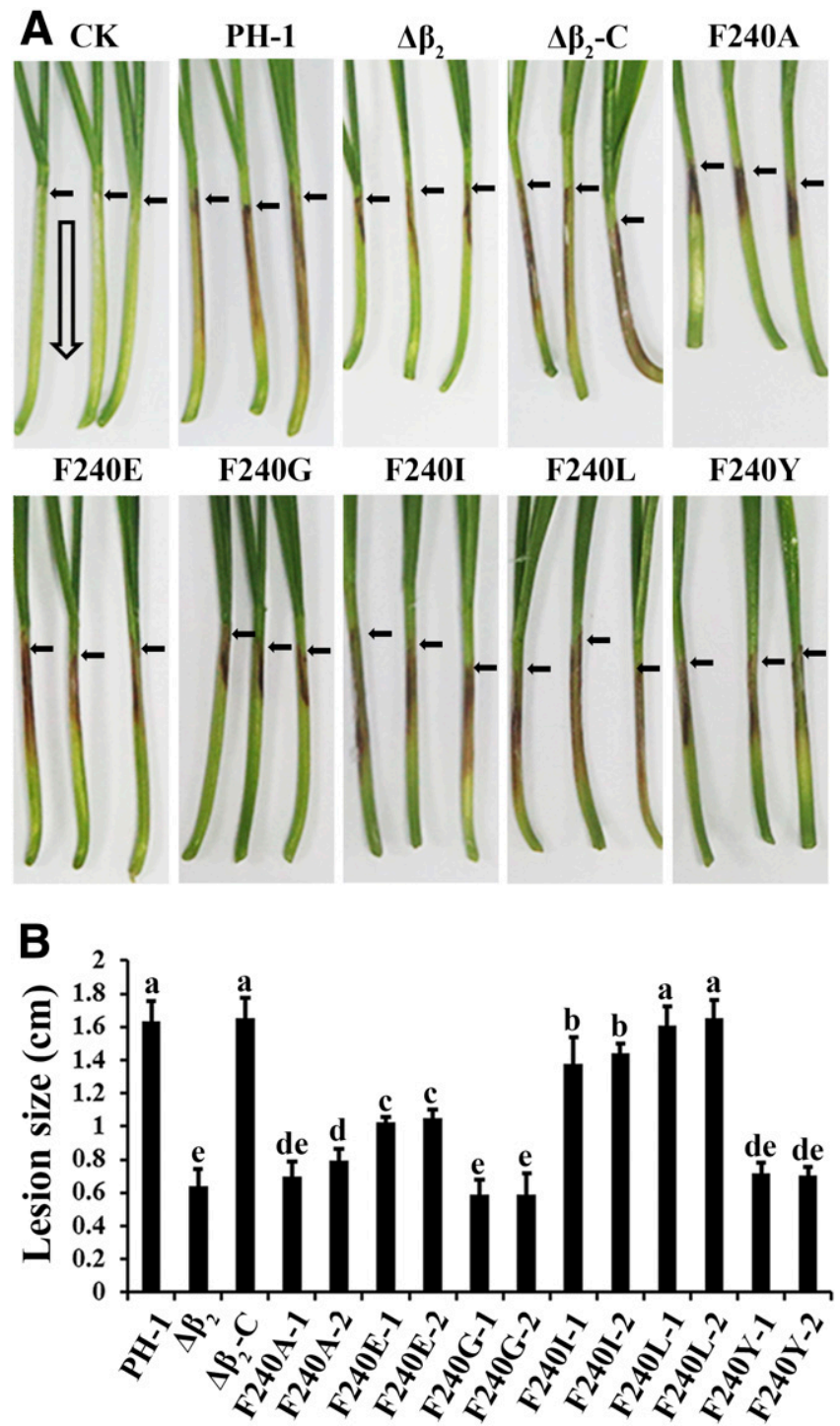

Fig. 5. Virulence of wild-type strain PH-1, $F g \beta_{2}$-tubulin deletion mutant $\Delta \beta_{2}$, the complemented mutant $\Delta \beta_{2}-\mathrm{C}$, and position 240 mutants. $\mathbf{A}$, Infected wheat coleoptiles were photographed at 10 days after inoculation. The small solid arrows indicate the inoculation sites and the hollow arrow indicates the lesion development direction. B, Lengths of brown lesions of wheat coleoptiles. Values are means \pm standard deviation of three repeated experiments. Means in a column with the same letter are not significantly different $(P<0.05)$.

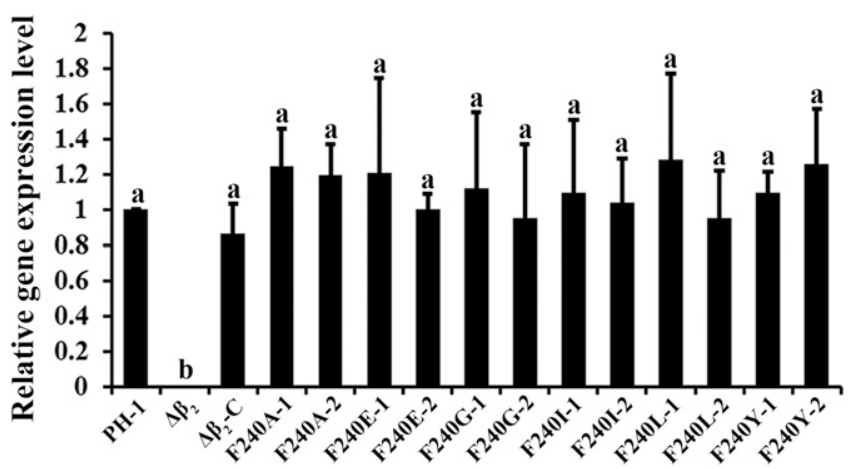

Fig. 6. Relative expression levels of $F g \beta_{2}$-tubulin gene of wild-type strain $\mathrm{PH}$ $1, F g \beta_{2}$-tubulin deletion mutant $\Delta \beta_{2}$, the complemented mutant $\Delta \beta_{2}-\mathrm{C}$ and position 240 mutants. Values are the means and standard deviation of three repeated experiments. Means of the column with the same letter are not significantly different $(P<0.05)$. 
morphology (Belvindrah et al. 2017; Huffaker et al. 1988; Niwa et al. 2013).

We also found that the F240A and F240G grew slower than the $\mathrm{Fg} \beta_{2}$-tubulin gene deletion mutant $\Delta \beta_{2}$ (Table 2). This may also be explained by disordered microtubule dynamics. The microtubule components differ in position-240 and $\Delta \beta_{2}$ mutants. The former contains $\alpha_{1^{-}}, \alpha_{2^{-}}, \beta_{1^{-}}$, and $\beta_{2} \mathrm{~F} 240 \mathrm{~A}-$, or $\beta_{2} \mathrm{~F} 240 \mathrm{G}-$ tubulin, while the latter contains $\alpha_{1^{-}}, \alpha_{2^{-}}$, and $\beta_{1}$-tubulin (Zhao et al. 2015). Our data suggest that the F240A and F240G mutations in $F g \beta_{2}$-tubulin might be more harmful to microtubule dynamics than the deletion of the $\mathrm{Fg} \beta_{2}$-tubulin and explained the reduced growth rate for the F240A and F240G mutants.

Ile and Tyr are similar to Leu and Phe, and we found that the hyphal morphology and microtubule polymerization of the F240Y and F240I mutants were normal (Figs. 2 and 7). However, their growth rates were significantly slower than those of the wild type or the F240L mutant (Table 2). The growth rate of F240Y was about $1 \mathrm{~cm} /$ day, which was slightly greater than that of the $F g \beta_{2}$-tubulin deletion mutant $\Delta \beta_{2}$. The F240I mutants grew at the similar rate as the F240L mutant but grew faster than the F240A, F240E, F240G, and F240Y mutants. These results indicate that position 240 is conserved and cannot be substituted with the other amino acids. Even a small change in the side chain of position 240 of $\mathrm{Fg}_{2^{-}}$ tubulin (F240I and F240Y) can be deleterious.

Although position 240 mutations disrupted the function of $\mathrm{Fg}_{2^{-}}$ tubulin and affected vegetative growth and hyphal morphology, conidial morphology was similar to those of the wild type and $\Delta \beta_{2^{-}}$ $\mathrm{C}$ and conidial production of all position 240 mutants was only decreased slightly (Figs. 3 and 4B). According to a previous study, the $F g \beta_{2}$-tubulin gene is vital to normal conidial morphology and

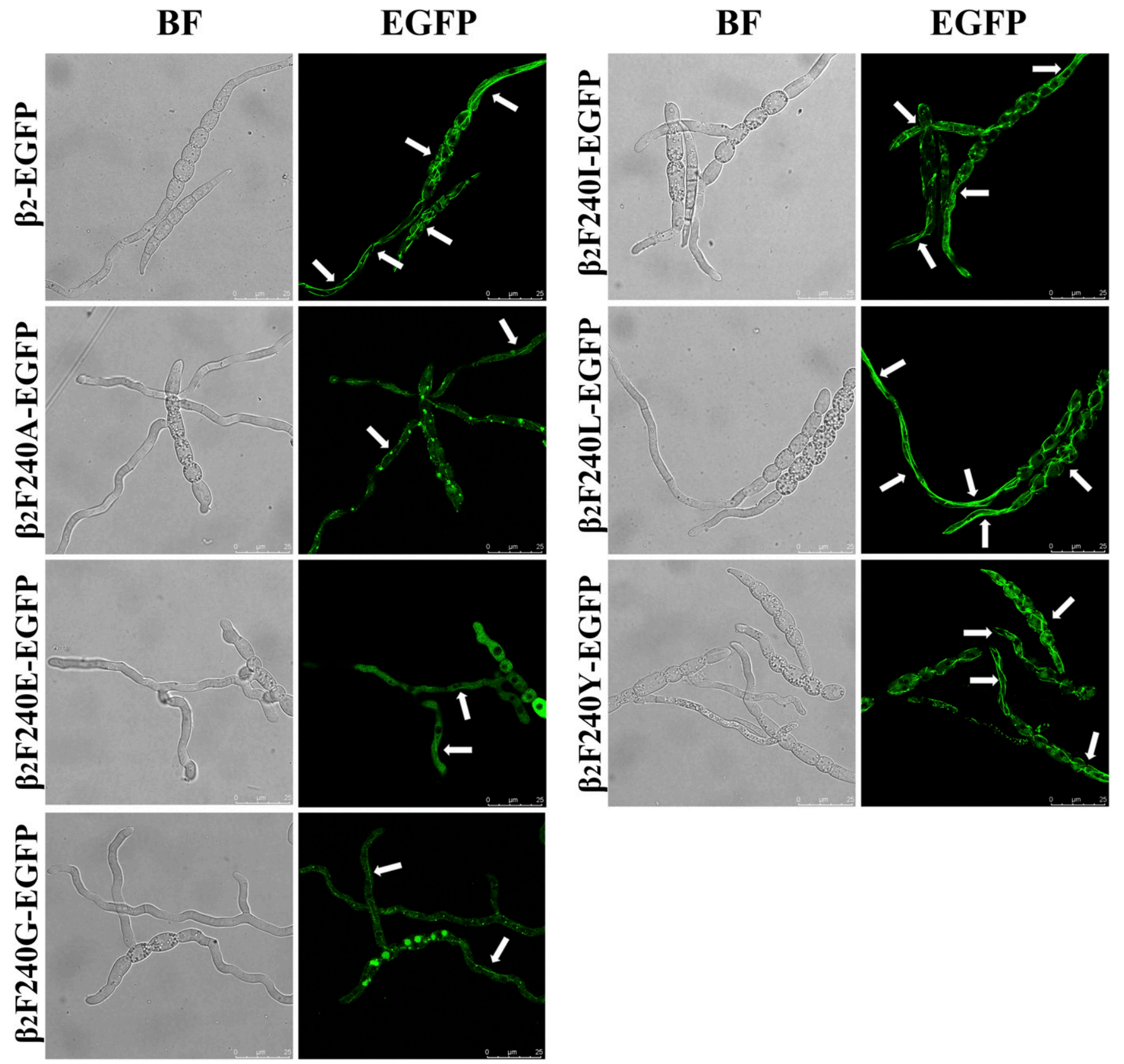

Fig. 7. Microtubule networks in $\beta_{2}$-tubulin-EGFP, $\beta_{2}$ F240A-EGFP, $\beta_{2}$ F240E-EGFP, $\beta_{2}$ F240G-EGFP, $\beta_{2}$ F240I-EGFP, $\beta_{2}$ F240L-EGFP, and $\beta_{2}$ F240Y-EGFP mutants. Fresh spores $\left(10^{5}\right)$ of all EGFP-tagging mutants were first incubated into $20 \mathrm{ml}$ of yeast extract-peptone-dextrose medium on an orbital shaker (175 rpm) at $25^{\circ} \mathrm{C}$ to germinate for $10 \mathrm{~h}$ and then spotted $5 \mu \mathrm{l}$ of culture onto the slides for micro-examination. The small white arrows indicate the microtubules in the hyphae. Bar $=25 \mu \mathrm{m}$. 
production, because mutants with $F g \beta_{2}$-tubulin gene deletion and interference had smaller conidia and significantly decreased conidial production than the wild type (Gu et al. 2019). Our results therefore indicate that mutations of position 240 of $\mathrm{Fg}_{2}$-tubulin and deletion of $F g \beta_{2}$-tubulin differ in their effects on conidiation. Although position 240 mutations cause serious defects in growth rate and hyphal morphology, they do not appear to affect conidiation.

As expected, all mutants except F240L had significantly decreased pathogenicity (Fig. 5). This was consistent with a recent study, which reported that $F g \beta_{2}$-tubulin gene deletion and interference mutants had decreased growth rates and pathogenicity (Gu et al. 2019). Microtubules are required for vesicle transport (Baravalle et al. 2005), and most pathogenic fungi rely on vesicle transport for extracellular secretion of virulence factors (Chanda et al. 2009; Conesa et al. 2001). The disordered microtubule dynamics in position 240 mutants may therefore affect the transport of virulence factors and contribute to the decreased pathogenicity. It is possible that decreased pathogenicity could reduce the survival of position 240 mutants.

We found that the F240A, F240E, F240I, F240L, and F240Y mutants had increased sensitivity to MBC (Table 2), suggesting that the substitution of Phe in position 240 of $F g \beta_{2}$-tubulin with other amino acids confers hypersensitivity to MBC. In previous studies, an altered expression level of $\beta$-tubulin affected the sensitivity of tubulin-binding agents (Kanakkanthara et al. 2011), and a decrease in the $F g \beta_{2}$-tubulin expression level increased the sensitivity to MBC (Gu et al. 2019). However, we found no significant difference between the expression levels of position 240 mutants and those of the wild-type strain PH-1 (Fig. 6). Other studies showed that the sensitivity of benzimidazole fungicide was determined by the binding affinity to the tubulin (Davidse and Flach 1977; Hollomon et al. 1998). We therefore performed molecular docking assays to determine whether the binding affinity of $\mathrm{MBC}$ to $\mathrm{Fg} \beta_{2}$-tubulin was changed by the mutations. The results indicate that F240A, F240E, F240I, F240L, and F240Y mutations increased the binding affinity between $\mathrm{Fg}_{2}$-tubulin and $\mathrm{MBC}$, which was consistent with the results of our MBC sensitivity assay (Tables 2 and 3). These findings suggest that these mutants, should they emerge within the population, would not survive under the selective pressure of MBC. Although the F240G mutant had a slightly decreased sensitivity to $\mathrm{MBC}$, its $\mathrm{EC}_{50}$ value of $0.8681 \pm 0.1635 \mu \mathrm{g} / \mathrm{ml}$ is still far below the dose used in the field. In addition, we found that the F240I mutant grew faster than all of the other mutants except F240L, but was even more sensitive to MBC than the F240L mutant, which means that the F240I mutant is not competitive than the wild type, or the F240L mutant.

In summary, most of the position 240 mutants had abnormal phenotypes including reduced growth rates, twisted hyphae, reduced pathogenicity, increased MBC sensitivity, and incomplete microtubule polymerization. These characteristics are detrimental to survival in the field. Although the F240A mutant had a slightly

TABLE 3. Binding free energy of wild-type and position 240-mutant Fusarium graminearum $\beta_{2}$-tubulins to methylbenzimidazol-2-ylcarbamate

\begin{tabular}{lc}
\hline Mutations & Binding free energy $(\mathrm{kcal} / \mathrm{mol})$ \\
\hline WT-F240 & -5.81 \\
F240A & -5.95 \\
F240E & -6.31 \\
F240G & -5.73 \\
F240I & -6.34 \\
F240L & -6.27 \\
F240Y & -5.94 \\
\hline
\end{tabular}

\section{Models Alignment}
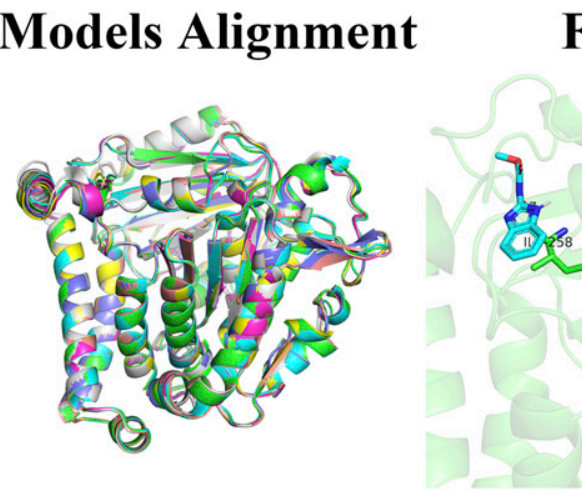

F240A

F240I

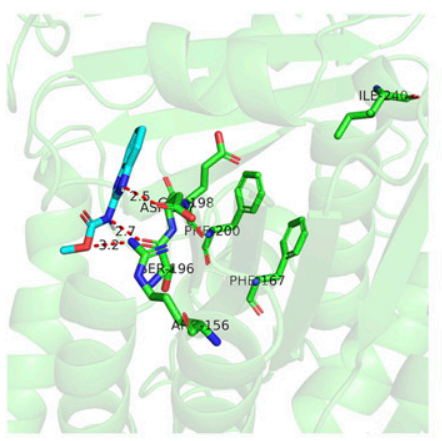

\section{(240}
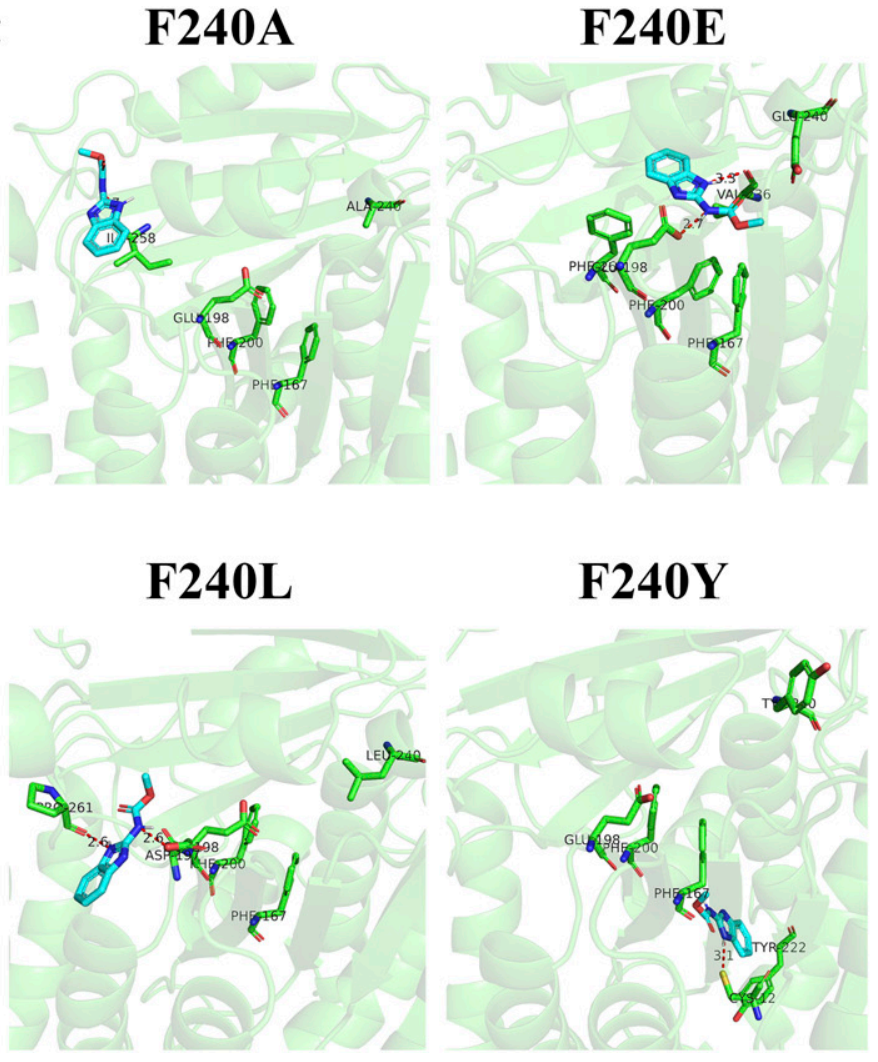

F240Y

F240L

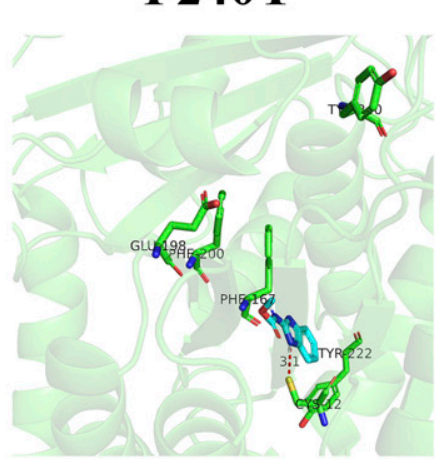

F240G

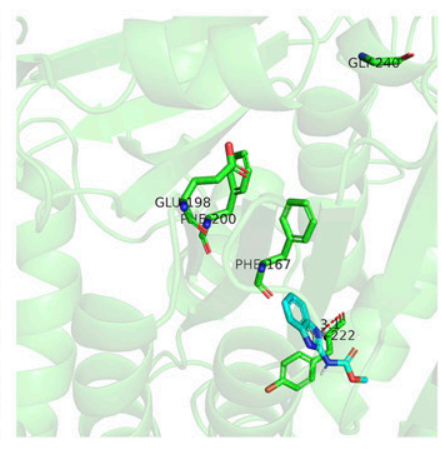

Fig. 8. Alignment of all $F g \beta_{2}$-tubulin models and molecular docking of methylbenzimidazol-2-ylcarbamate (MBC) to $F g \beta_{2}$-tubulin. Homology models of wildtype and position 240 mutant $F g \beta_{2}$-tubulins were constructed using the online server at I-TASSER based on the same remark parents (PDB ID: 3hke_D, 1tvk_B, 3cb2_A, 4u3j_B, and 2btq_A). Molecular docking was performed using AutoDockTools in MGLTools software (version, 1.5.6) with semiflexible docking. The Hbond has been remarked by dashed lines with numbers. 
decreased sensitivity to $\mathrm{MBC}$, its decreased growth rate and pathogenicity indicate that F240A is unlikely to survive and compete with the wild-type in the field. Thus, F240 is vital to the structure and functions of $F g \beta_{2}$-tubulin and cannot be substituted by other amino acids. The results reveal the uniqueness of F240 of $\mathrm{Fg}_{2}$-tubulin and increase our understanding of the function of $\mathrm{Fg}_{2}$-tubulin in $\mathrm{F}$. graminearum and of tubulins in other filamentous fungi. These results could also be useful for optimizing and developing tubulin-binding fungicides.

\section{LITERATURE CITED}

Aher, A., and Akhmanova, A. 2018. Tipping microtubule dynamics, one protofilament at a time. Curr. Opin. Cell Biol. 50:86-93.

Albertini, C., Gredt, M., and Leroux, P. 1999. Mutations of the $\beta$-tubulin gene associated with different phenotypes of benzimidazole resistance in the cereal eyespot fungi Tapesia yallundae and Tapesia acuformis. Pestic. Biochem. Physiol. 64:17-31.

Bai, G., and Shaner, G. 2004. Management and resistance in wheat and barley to Fusarium head blight. Annu. Rev. Phytopathol. 42:135-161.

Baravalle, G., Schober, D., Huber, M., Bayer, N., Murphy, R. F., and Fuchs, R. 2005. Transferrin recycling and dextran transport to lysosomes is differentially affected by bafilomycin, nocodazole, and low temperature. Cell Tissue Res. 320:99-113.

Belvindrah, R., Natarajan, K., Shabajee, P., Bruel-Jungerman, E., Bernard, J., Goutierre, M., Moutkine, I., Jaglin, X. H., Savariradjane, M., Irinopoulou, T., Poncer, J., Janke, C., and Francis, F. 2017. Mutation of the $\alpha$-tubulin Tuba1a leads to straighter microtubules and perturbs neuronal migration. J. Cell Biol. 216:2443-2461.

Bruno, K. S., Tenjo, F., Li, L., Hamer, J. E., and Xu, J. R. 2004. Cellular localization and role of kinase activity of PMK1 in Magnaporthe grisea. Eukaryot. Cell 3:1525-1532.

Chanda, A., Roze, L. V., Kang, S., Artymovich, K. A., Hicks, G. R., Raikhel, N. V., Calvo, A. M., and Linz, J. E. 2009. A key role for vesicles in fungal secondary metabolism. Proc. Natl. Acad. Sci. USA 106:19533-19538.

Chen, C. J., Yu, J. J., Bi, C. W., Zhang, Y. N., Xu, J. Q., Wang, J. X., and Zhou, M. G. 2009. Mutations in a $\beta$-tubulin confer resistance of Gibberella zeae to benzimidazole fungicides. Phytopathology 99:1403-1411.

Chen, Y., Wang, J., Yang, N., Wen, Z. Y., Sun, X. P., Chai, Y. R., and Ma, Z. H. 2018. Wheat microbiome bacteria can reduce virulence of a plant pathogenic fungus by altering histone acetylation. Nat. Commun. 9:3429.

Conesa, A., Punt, P. J., Van Luijk, N., and Van den Hondel, C. A. M. J. J. 2001. The secretion pathway in filamentous fungi: A biotechnological view. Fungal Genet. Biol. 33:155-171.

Davidse, L. C. 1986. Benzimidazole fungicides: Mechanism of action and biological impact. Annu. Rev. Phytopathol. 24:43-65.

Davidse, L. C., and Flach, W. 1977. Differential binding of methyl benzimidazol2-yl carbamate to fungal tubulin as a mechanism of resistance to this antimitotic agent in mutant strains of Aspergillus nidulans. J. Cell Biol. 72:174-193.

Gu, K. X., Song, X. S., Xiao, X. M., Duan, X. X., Wang, J. X., Duan, Y. B., Hou, Y. P., and Zhou, M. G. 2019. A $\beta_{2}$-tubulin dsRNA derived from Fusarium asiaticum confers plant resistance to multiple phytopathogens and reduces fungicide resistance. Pestic. Biochem. Physiol. 153:36-46.

Hari, M., Wang, Y. Q., Veeraraghavan, S., and Cabral, F. 2003. Mutations in $\alpha-$ and $\beta$-tubulin that stabilize microtubules and confer resistance to colcemid and vinblastine. Mol. Cancer Ther. 2:597-605.

Hollomon, D. W., Butters, J. A., Barker, H., and Hall, L. 1998. Fungal $\beta$-tubulin, expressed as a fusion protein, binds benzimidazole and phenylcarbamate fungicides. Antimicrobiol. Agents Chemother. 42:2171-2173.

Huffaker, T. C., Thomas, J. H., and Botstein, D. 1988. Diverse effects of $\beta$-tubulin mutations on microtubule formation and function. J. Cell Biol. 106:1997-2010.

Jia, L. J., Tang, H. U., Wang, W. Q., Yuan, T. L., Wei, W. Q., Pang, B., Gong, X. M., Wang, S. F., Li, Y. J., Zhang, D., Liu, W., and Tang, W. H. 2019. A linear nonribosomal octapeptide from Fusarium graminearum facilitates cell-to-cell invasion of wheat. Nat. Commun. 10:922.
Kanakkanthara, A., Wilmes, A., O'Brate, A., Escuin, D., Chan, A., Gjyrezi, A., Crawford, J., Rawson, P., Kivell, B., Northcote, P. T., Hamel, E., Giannakakou, P., and Miller, J. H. 2011. Peloruside- and laulimalide-resistant human ovarian carcinoma cells have $\beta \mathrm{I}$-tubulin mutations and altered expression of $\beta \mathrm{II}-$ and $\beta \mathrm{III}-$ tubulin isotypes. Mol. Cancer Ther. 10:1419-1429.

Kavallaris, M., Kuo, D. Y. S., Burkhart, C. A., Regl, D. L., Norris, M. D., Haber, M., and Horwitz, S. B. 1997. Taxol-resistant epithelial ovarian tumors are associated with altered expression of specific $\beta$-tubulin isotypes. J. Clin. Invest. 100:1282-1293.

Li, H. X., Lu, Y. J., Wang, J. X., and Zhou, M. G. 2002. Comparison of mutations in the $\beta$-tubulin gene that confer resistance to $\mathrm{MBC}$ in four plant pathogenic fungi. J. Nanjing Agric. Univ. 25:41-44.

Liu, S. M., Duan, Y. B., Ge, C. Y., Chen, C. J., and Zhou, M. G. 2013. Functional analysis of the $\beta 2$-tubulin gene of Fusarium graminearum and the $\beta$-tubulin gene of Botrytis cinerea by homologous replacement. Pest Manag. Sci. 69:582-588.

Ma, Z. H., Yoshimura, M. A., Holtz, B. A., and Michailides, T. J. 2005. Characterization and PCR-based detection of benzimidazole-resistant isolates of Monilinia laxa in California. Pest Manag. Sci. 61:449-457.

Niwa, S., Takahashi, H., and Hirokawa, N. 2013. $\beta$-Tubulin mutations that cause severe neuropathies disrupt axonal transport. EMBO J. 32:1352-1364.

Nogales, E. 2000. Structural insights into microtubule function. Annu. Rev. Biochem. 69:277-302.

Qiu, J. B., Huang, T. T., Xu, J. Q., Bi, C. W., Chen, C. J., and Zhou, M. G. 2012. $\beta$-Tubulins in Gibberella zeae: Their characterization and contribution to carbendazim resistance. Pest Manag. Sci. 68:1191-1198.

Reijo, R. A., Cooper, E. M., Beagle, G. J., and Huffaker, T. C. 1994. Systematic mutational analysis of the yeast $\beta$-tubulin gene. Mol. Biol. Cell 5:29-43.

Roy, A., Kucukural, A., and Zhang, Y. 2010. I-TASSER: A unified platform for automated protein structure and function prediction. Nat. Protoc. 5:725-738.

Steinmetz, M. O., and Prota, A. E. 2018. Microtubule-targeting agents: Strategies to hijack the cytoskeleton. Trends Cell Biol. 28:776-792.

Ti, S. C., Pamula, M. C., Howes, S. C., Duellberg, C., Cade, N. I., Kleiner, R. E., Forth, S., Surrey, T., Nogales, E., and Kapoor, T. M. 2016. Mutations in human tubulin proximal to the kinesin-binding site alter dynamic instability at microtubule plus- and minus-ends. Dev. Cell 37:72-84.

Tischfield, M. A., Baris, H. N., Wu, C., Rudolph, G., Maldergem, L. V., $\mathrm{He}$, W., et al. 2010. Human TUBB3 mutations perturb microtubule dynamics, kinesin interactions, and axon guidance. Cell 140:74-87.

Vitre, B., Coquelle, F. M., Heichette, C., Garnier, C., Chrétien, D., and Arnal, I. 2008. EB1 regulates microtubule dynamics and tubulin sheet closure in vitro. Nat. Cell Biol. 10:415-421.

Yarden, O., and Katan, T. 1993. Mutations leading to substitutions at amino acids 198 and 200 of beta-tubulin that correlate with benomyl resistance phenotypes of field strains of Botrytis cinerea. Mol. Plant Pathol. 83:1478-1483.

Zhang, X. W., Jia, L. J., Zhang, Y., Jiang, G., Li, X., Zhang, D., and Tang, W. H. 2012. In planta stage-specific fungal gene profiling elucidates the molecular strategies of Fusarium graminearum growing inside wheat coleoptiles. Plant Cell 24:5159-5176.

Zhang, Y. 2008. I-TASSER server for protein 3D structure prediction. BMC Bioinform. 9:40.

Zhang, Y., Gao, T., Shao, W. Y., Zheng, Z. T., Zhou, M. G., and Chen, C. J. 2016. The septins FaCdc 3 and $\mathrm{FaCdc} 12$ are required for cytokinesis and affect asexual and sexual development, lipid metabolism and virulence in Fusarium asiaticum. Mol. Plant Pathol. 18:1282-1294.

Zhang, Z. X., Gao, B. B., He, Z. Z., Li, L. S., Shi, H. Y., and Wang, M. H. 2019. Enantioselective metabolism of four chiral triazole fungicides in rat liver microsomes. Chemosphere 224:77-84.

Zhao, Z. T., Liu, H. Q., Luo, Y. P., Zhou, S. Y., An, L., Wang, C. F., Jin, Q. J., Zhou, M. G., and Xu, J. R. 2015. Molecular evolution and functional divergence of tubulin superfamily in the fungal tree of life. Sci. Rep. 4:6746.

Zheng, Z. T., Gao, T., Zhang, Y., Hou, Y. P., Wang, J. X., and Zhou, M. G. 2014. FgFim, a key protein regulating resistance to the fungicide JS399-19, asexual and sexual development, stress responses and virulence in Fusarium graminearum. Mol. Plant Pathol. 15:488-499.

Zhu, Y. Y., Liang, X. Y., Li, Y. J., Duan, Y. B., Zheng, Z. T., Wang, J. X., and Zhou, M. G. 2018. F240 of $\beta_{2}$-tubulin explains why Fusarium graminearum is less sensitive to carbendazim than Botrytis cinerea. Phytopathology 108:352-361. 